\title{
SHADOW ECONOMY, ECONOMIC GROWTH AND LABOR MARKET. ROMANIAN CASE
}

\author{
Associate Professor PhD Mutaşcu Mihai, West University of Timisoara, \\ mihai.mutascu@gmail.com
}

\begin{abstract}
The shadow economy is represented by the whole economic activities which are realized at the border of the criminal laws, social laws or fiscal laws or which are skipping (massive) from the inventory of national accounts. The paper is intended to quantify and analyze the impact of the shadow economy on the main variables that may characterize economic growth and labor market, for the Romanian case.
\end{abstract}

Keywords: shadow economy, effects, economic growth, labor market

JEL codes: O17, O40, J40

The paper is intended to quantify and analyze the impact of the shadow economy (S) on the main variables that may characterize economic growth and labor market, for the Romanian case. The economic growth is being treated through gross domestic product (GDP) as an essential variable for measuring economic growth, and labor market is treated through unemployment rate (RS) and net average monthly salary (SNL).

The shadow economy values are taken from studies made by Friedrich Schneider, specifically from "Dimensions of the Shadow Economy", published in The Independent Review, VV, n.1, 2000, as well as from "Shadow Economies of 145 Countries all over the World: Estimation Results over the period 1999 to 2003 ", Working Paper No. 2005-13, Center for Research in Economics, Management and the Arts (CREMA), Basel, March 2007.

The analysis of the impact of the shadow economy on economic growth and labor market is made for the $1990-2007^{1}$ years, watching the effects of the shadow economy on gross domestic product, unemployment rate and average net monthly salary. The analysis method used is the econometric modeling, by using the software package EViews $5.0^{2}$.

Accordingly, we have built three one-variables regression models like:

$$
Y_{t}=\alpha+\beta_{i} x X_{i t}+\varepsilon_{t}
$$

where

- $Y_{t}$ is the dependent variable;

- $\alpha$ the intercept coefficient;

- $\beta_{i}$ the slope coefficients;

- $\mathrm{X}_{\mathrm{it}}$ the independent variable;

- $\varepsilon_{t}$ the random variable;

- $i$ the number of the variables;

- $t$ the time interval (1990-2007).

\footnotetext{
${ }^{1}$ Time series processed from the Annual Report of National Bank of Romania 1993-2007 and from the Romanian Statistical Yearbook 1990-2007.

${ }^{2}$ Copyright (C) 1994-2004 Quantitative Micro Software, LLC, All Rights Reserved.
} 
Concretely, it will be looked up for quantifying the relationship that exists between the shadow economy level and the GDP volume, the unemployment rate and average net monthly wage.

The time series are illustrated in Table 1.

Table no.1

Shadow economy level, GDP volume, unemployment rate and medium net monthly wages, in Romania, in 1990-2007 period

\begin{tabular}{|c|c|c|c|c|}
\hline Year & $\begin{array}{c}\text { Shadow economy } \\
\text { level }\end{array}$ & GDP & $\begin{array}{c}\text { Unemployment } \\
\text { rate }\end{array}$ & $\begin{array}{c}\text { Medium net monthly } \\
\text { wages }\end{array}$ \\
\hline u.m & \% of GDP & Mil. Ron & \% & Ron \\
\hline 1990 & 18 & 0.09 & 1.6 & 0.70 \\
\hline 1991 & 16 & 0.22 & 3 & 1.18 \\
\hline 1992 & 16 & 0.60 & 8.2 & 3.26 \\
\hline 1993 & 16 & 2.00 & 10.4 & 10.13 \\
\hline 1994 & 18.3 & 4.98 & 10.9 & 19.85 \\
\hline 1995 & 18.3 & 7.21 & 9.5 & 28.30 \\
\hline 1996 & 19.3 & 10.89 & 6.6 & 43.37 \\
\hline 1997 & 19.3 & 25.3 & 8.9 & 94.05 \\
\hline 1998 & 19.3 & 37.38 & 10.4 & 136.03 \\
\hline 1999 & 34.4 & 54.57 & 11.8 & 199.01 \\
\hline 2000 & 34.4 & 80.38 & 10.5 & 291.16 \\
\hline 2001 & 36.1 & 116.77 & 8.8 & 365.97 \\
\hline 2002 & 36.1 & 151.48 & 8.4 & 452.57 \\
\hline 2003 & 37.4 & 197.56 & 7.4 & 565.81 \\
\hline 2004 & 37.4 & 246.47 & 6.3 & 687.51 \\
\hline 2005 & 26.8 & 288.05 & 5.9 & 848.00 \\
\hline 2006 & 26.8 & 344.54 & 5.2 & $1,099.00$ \\
\hline 2007 & 26.8 & 404.71 & 4.57 & $1,266.00$ \\
\hline
\end{tabular}

Source : data taken from Friedrich Schneider $(2000,2007)$ and from the Annual Report of National Bank of Romania, 1993-2007, and also from the Romanian Statistical Yearbook 1990-2007

1. The modeling of the impact of show economy on gross domestic product is based on a one-variable regressive model like:

$$
Y_{t}=\alpha+\beta_{t} x X_{t}+\varepsilon_{t}
$$

where $Y_{t}$ is the dependent variable - GDP (gross domestic product), $\alpha$ the intercept coefficient, $\beta$ the slope coefficients, $X_{t}$ the independent variable - $S$ (the shadow economy level, as a weight of GDP), $\varepsilon_{t}$ the random variable, $i$ the number of the variables - 1, and $t$ the time interval (19902007).

The mathematical model will be:

$$
\text { P.I.B. }=\alpha+\beta x S+\varepsilon_{t}
$$

After modeling the two time series, the results obtained shall become (Table 2): 


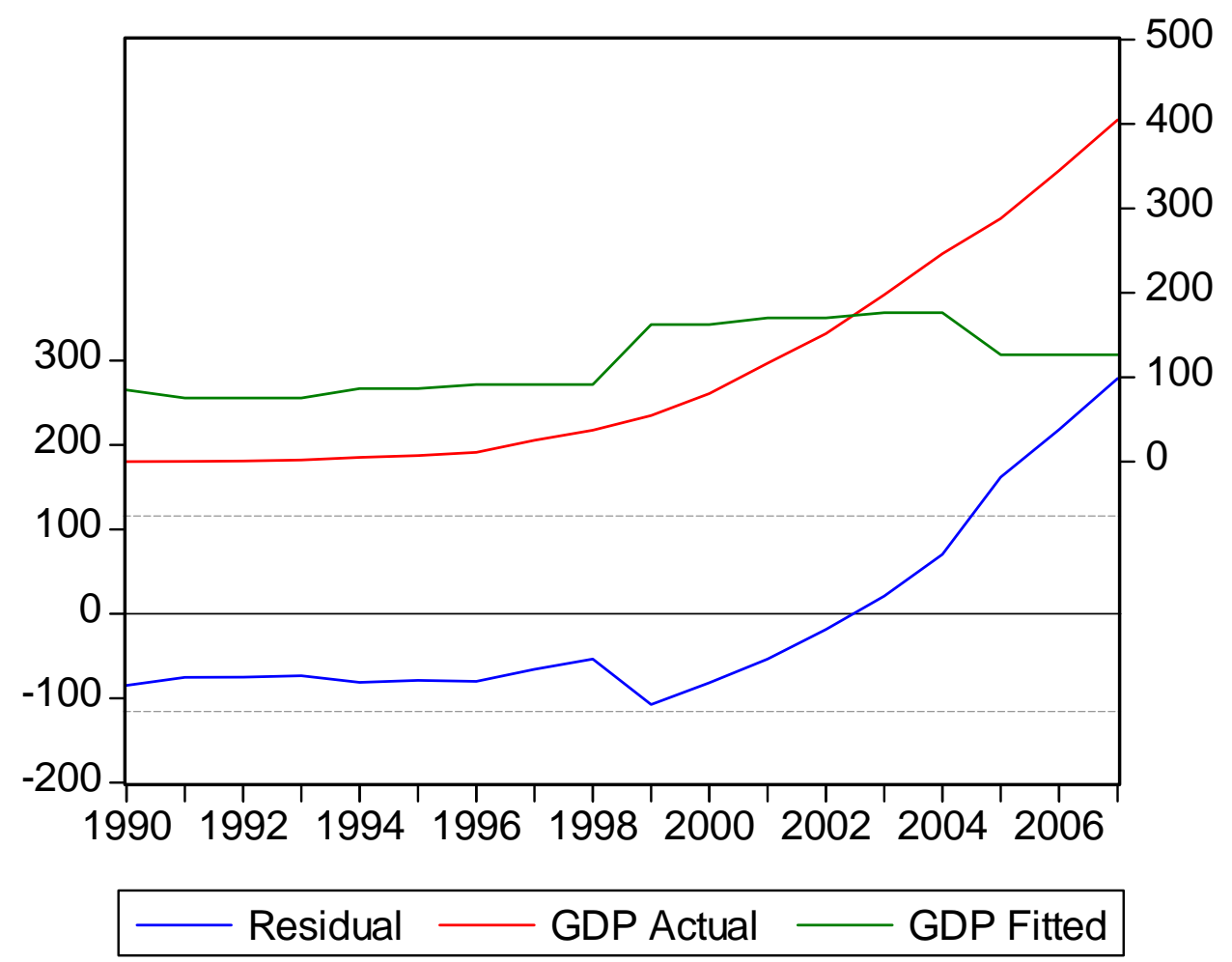

Fig. no. 1 - The trend of GDP, estimated GDP with the model and the residual values, in Romania, for the time interval 1990-2007

2. The modeling of the impact of show economy on unemployment rate is based on a onevariable regressive model like:

$$
Y_{t}=\alpha+\beta_{t} x X_{t}+\varepsilon_{t}
$$

where $Y_{t}$ is the dependent variable - RS (unemployment rate), $\alpha$ the intercept coefficient, $\beta$ the slope coefficients, $X_{t}$ the independent variable - $S$ (the shadow economy level, as a weight of GDP), $\varepsilon_{t}$ the random variable, $i$ the number of the variables - 1, and $t$ the time interval (19902007).

The model will be:

$$
R S=\alpha+\beta x S+\varepsilon_{t}
$$

After modeling the two time series, the results obtained shall be (Table 3): 
Table no. 3

Statistical tests related to the modelling of the shadow economy level impact

on the unemployment rate

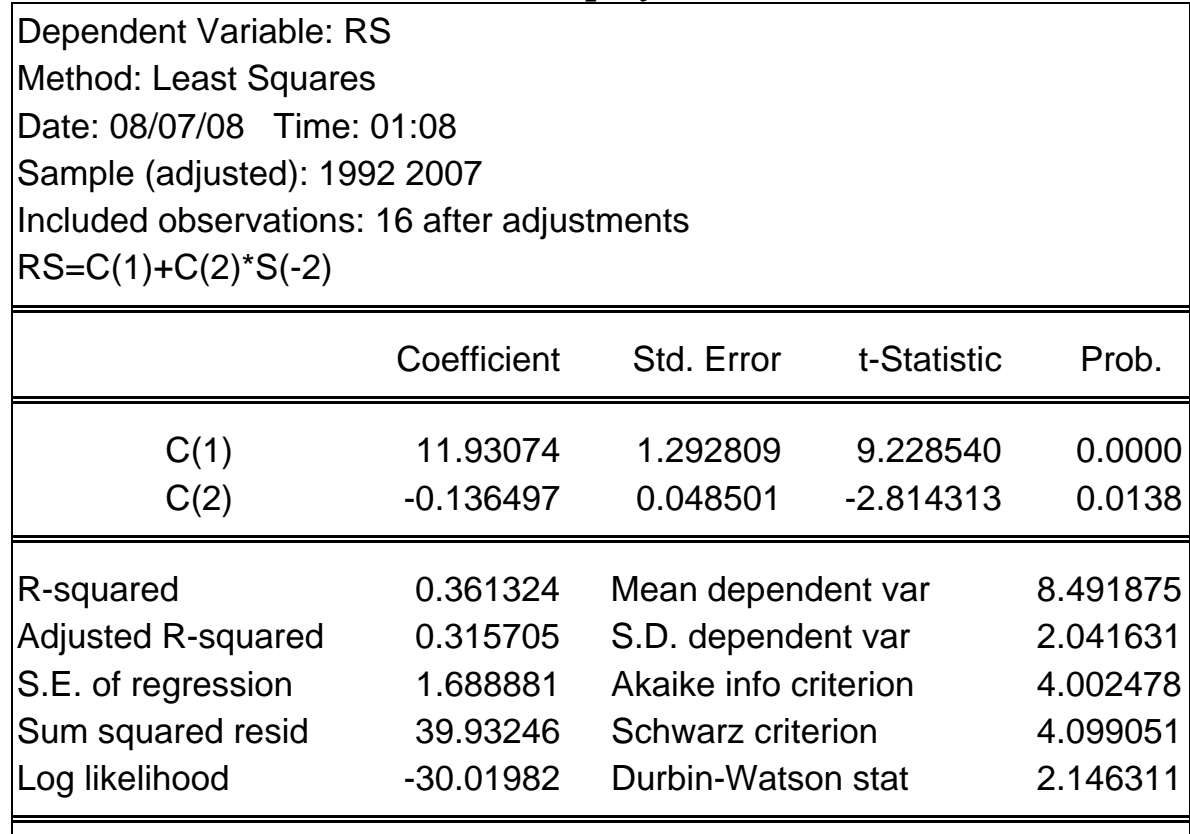

Analyzing the data from Table 3, we can draw the following conclusions:

- the values of standard errors of the regression coefficient are inferior to the value of the coefficient, taken as a module, which strengthens the validity of their estimation, sustained also by small value of probability;

- the correlation coefficient, with a value of $36,13 \%$ shows that the statistical relation between the dependent variable - RS and the endogen variable $-\mathrm{S}$ is relatively moderate; the changes of the shadow economy are included in an appreciable proportion in the changes of the unemployment rate;

- the Durbin-Watson test, with a value slightly above the critical threshold 2, indicates that the residual variables are not auto-correlated

We may appreciate that the model shall be considered representative for the description of the linkage between shadow economy level and unemployment rate at a macro economic level.

As a result, the model can be written:

$$
\mathrm{RS}=11,9307-0,136497 \times \mathrm{S}
$$

Analyzing this model we may say that, in the Romanian case, the shadow economy has a moderate impact over the unemployment rata, an increase of 1\% determines a decrease of 0,13\% for the unemployment rata. This is fully evident after about 2 years because the shadow economy behaves like an "absorber" on the labor market, so the deficit offer of employment compensated by illegal employment created by shadow economy(illegal work).

The trends of historical RS, estimated RS with the model and the residual values, in Romania's case for the time interval 1990-2007are presented in Figure 2. 


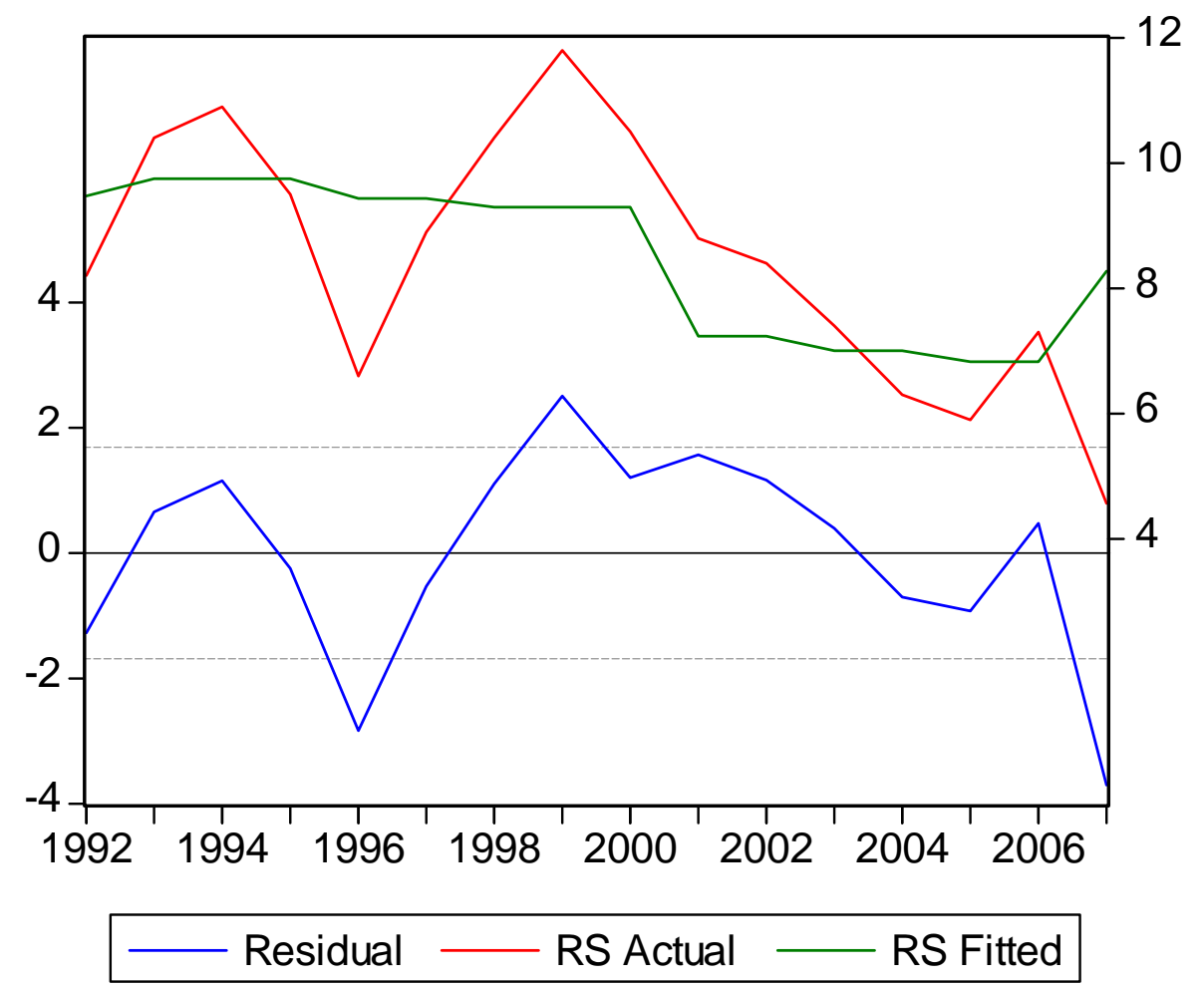

Fig. no. 2 - The trend of $R S$, estimated $R S$ with the model and the residual values, in Romania, for the time interval 1990-2007

3. The modeling of the impact of show economy on net average monthly salary is based on a one-variable regressive model like:

$$
Y_{t}=\alpha+\beta_{t} x X_{t}+\varepsilon_{t}
$$

where $Y_{t}$ is the dependent variable - SNL (net average monthly salary), $\alpha$ the intercept coefficient, $\beta$ the slope coefficients, $X_{t}$ the independent variable - $S$ (the shadow economy level, as a weight of GDP), $\varepsilon_{t}$ the random variable, $i$ the number of the variables - 1, and $t$ the time interval (19902007).

The model will become:

$$
S N L=\alpha+\beta x S+\varepsilon_{t}
$$

After modeling the two time series, the results obtained shall be (Table 4): 
Table no. 4

Statistical tests related to the modelling of the shadow economy level impact

on the net average monthly salary

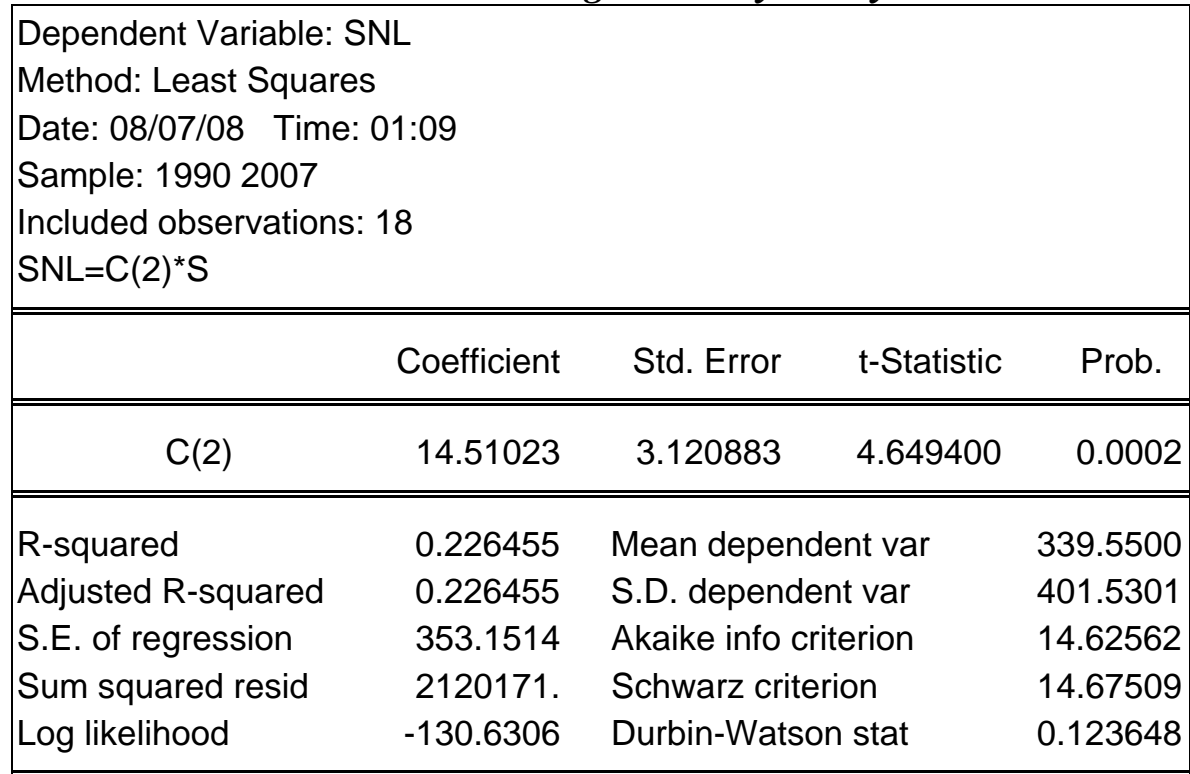

Like in the first model, the intercept coefficient - $\alpha$ was negative, so the regression was recast by exuding the intercept coefficient.

Analyzing the data from Table 4, we can draw the following conclusions:

- the values of standard errors of the regression coefficient are inferior to the value of the coefficient, taken as a module, which strengthens the validity of their estimation, sustained also by small value of probability;

- the correlation coefficient, with a value of $23,15 \%$ shows that the statistical relation between the dependent variable - SNL and the endogen variable -S is relatively small; the changes of the shadow economy are included in an diminished proportion in the changes of the net average monthly salary;

- the Durbin-Watson test, with a value slightly above the critical threshold 2, indicates that the residual variables are not auto-correlated.

Accordingly, we may appreciate that the model shall be considered representative for the description of the linkage between shadow economy level and net average monthly salary at a macro economic level.

As a result, the model can be written:

$$
\mathrm{SNL}=14,5102 \times \mathrm{S}
$$

Analyzing this model we may say that, in the Romanian case, the shadow economy has a moderate impact over the net average monthly salary, but a positive one, an increase of 1\% determines an increase of 14,5\% for the net average monthly salary. In other words, through new work places created by the shadow economy the illegal earnings are growing up which are revolved through the input.

The trends of historical SNL, estimated SNL with the model and the residual values, in Romania's case for the time interval 1990-2007 are presented in Figure 3. 


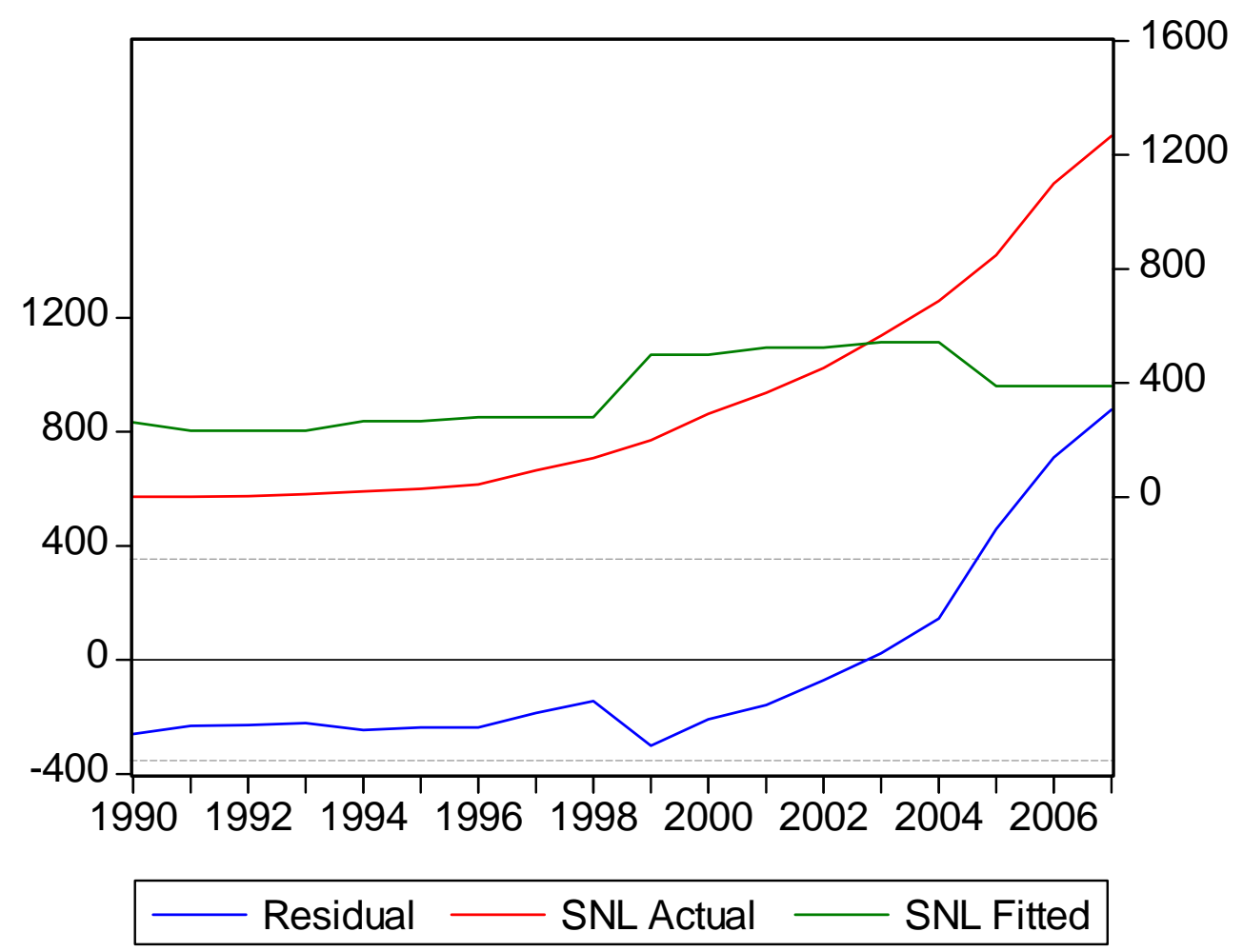

Fig. no. 3 - The trend of SNL, estimated SNL with the model and the residual values, in Romania, for the time interval 1990-2007

Analyzing the four models we may conclude that paradoxically the shadow economy has some visible effects over the legal economic and social life, the main ideas are presented next:

- the shadow economy has a low impact, but a benefic one, on the efficiency of the functioning of the economy, so an increase of its level with 1\% determines an increase of GDP with 4,7\%. In other properly, the shadow economy is a "necessary evil", its mechanisms support a legal economic growth of the country.

- the shadow economy has a moderate impact over the inflation rate, an increase of 1\% determines a decrease of 6,1\% for the inflation rate. In this case, the inflationary pressure is caused by the sustentation of the shadow economy for the goods and services offer on real market and is supplementing the legal offer (compensates the goods and services legal supply).

- the shadow economy has a moderate impact over the unemployment rata, an increase of 1\% determines a decrease of $0,13 \%$ for the unemployment rata. This is fully evident after about 2 years because the shadow economy behaves like an "absorber" on the labor market, so the deficit offer of employment compensated by illegal employment created by shadow economy(illegal work).

- the shadow economy has a moderate impact over the net average monthly salary, but a positive one, an increase of 1\% determines an increase of 14,5\% for the net average monthly salary. In other words, through new work places created by the shadow economy the illegal earnings are growing up which are revolved through the input.

\section{References:}

1. Del'Anno Roberto, (2003), Estimating the shadow economy in Italy: A structural equation approach, Discussion Paper, Department of Economics and Statistics, University of Salerno.

2. Feige Edgar, (2004), The underground economy and the currency enigma, Supplement to Public Finance/ Finances Publiques, No.49. 
3. Schneider Friedrich, (2007), Shadow Economies of 145 Countries all over the World: Estimation Results over the Period 1999 to 2003", Working Paper No. 2005-13, Center for Research in Economics, Management and the Arts (CREMA), Basel.

4. Schneider Friedrich, (2000), Dimensions of the Shadow Economy, The Independent Review, v.V, n.1. 